\title{
FATORES ASSOCIADOS À AUSÊNCIA DE CONTROLE GLICÊMICO EM DIABÉTICOS ATENDIDOS EM UNIDADES DE ATENÇÃO PRIMÁRIA À SAÚDE
}

\author{
Helena Fraga Maia*, Zeila Rocha Carmo** \\ Autor correspondente: Helena Fraga Maia. E-mail: helenafragamaia@gmail.com \\ * Professora Adjunta da Universidade do Estado da Bahia/. Doutorado em Saúde Pública pela Universidade Federal da \\ Bahia (2010). Atualmente vem desenvolvendo pesquisas no âmbito do Pós-Doutorado (ISC/UFBA). \\ ** Fisioterapeuta pela Universidade do Estado da Bahia.
}

\section{Resumo}

OBJETIVO: Investigar os fatores associados à ausência de controle glicêmico em pacientes com DM tipo 2. MATERIAL E MÉTODOS: Trata-se se um estudo descritivo de corte transversal conduzido com pacientes com diagnóstico de Diabetes Mellitus e que estavam em tratamento em unidades de saúde do Distrito Sanitário Cabula-Beiru. Considerou-se ausência de controle glicêmico os valores iguais ou acima de $126 \mathrm{mg} / \mathrm{dl}$ obtidos por exame de glicose plasmática no sangue do paciente em jejum e relatados pelos mesmos. A magnitude da associação entre as variáveis estudadas e a ausência de controle glicêmico foi estimada pelo cálculo da razão da Odds ratio, adotando-se o intervalo de confiança a $95 \%$ (IC95\%). Posteriormente, foram realizadas análises multivariadas utilizando-se a regressão logística, a partir de um modelo teórico definido a priori, discriminando os fatores de risco em blocos hierarquizados. O projeto de pesquisa foi aprovado pelo CEP/UNEB (241.434/2013) e financiado pelo PRO Saúde/PET-Saúde 2012. RESULTADOS: Participaram deste estudo 127 usuários que preencheram adequadamente os critérios de elegibilidade, dentre estes, 51 (40,2\%) apresentavam níveis glicêmicos considerados como não controlados. A ausência de controle glicêmico mostrou-se independentemente associada a não praticar atividade física leve a moderada regularmente $(O R=2,14$; IC95\%:1,88-2,22), mesmo após ajuste por sexo, renda, relativas a hábitos de vida e qualidade da assistência. CONCLUSÃO: O número elevado de indivíduos que negaram a pratica de atividade física associou-se significativamente a ausência de controle glicêmico. Este fator pode contribuir para o aumento das complicações cardiovasculares e para um prognóstico desfavorável em termos de agravos e óbitos.

Palavras-chave: Diabetes Mellitus; Controle Glicêmico; Estilo de Vida. 


\title{
FACTORS ASSOCIATED WITH LACK OF CONTROL IN DIABETIC GLYCEMIC SERVED IN PRIMARY HEALTH UNITS
}

\begin{abstract}
PURPOSE: To investigate the factors associated with lack of glycemic control in patients with type 2 DM. METHODS: This was a cross-sectional study conducted with patients diagnosed with Diabetes Mellitus and who were being treated in health units Cabula Beiru District Health. Considered the absence of glycemic control values equal to or above $126 \mathrm{mg} / \mathrm{dl}$ obtained by examination of plasma glucose in the patient's fasting blood and reported by them. The magnitude of the association between variables and the lack of glycemic control was estimated by calculating the ratio of the odds ratio, adopting the confidence interval of $95 \%$ (95\%). Later, there were multivariate analysis using logistic regression, from a theoretical model established in advance, specifying the risk factors in hierarchical blocks. The research project was approved by the CEP / UNEB (241 434/2013) and funded by the Health PRO / PET-Health 2012. RESULTS: The study included 127 users that adequately met the eligibility criteria, among these, 51 (40.2\%) had glucose levels considered uncontrolled. The lack of glycemic control was shown to be independently associated with not practicing mild to moderate physical activity regularly ( $\mathrm{OR}=2.14 ; 95 \% \mathrm{Cl}: 1.88$ to 2.22$)$, even after adjustment for gender, income, related to habits life and quality of care. CONCLUSION: The high number of individuals who denied the practice of physical activity was significantly associated with the absence of glycemic control. This factor may contribute to the increase in cardiovascular complications and an unfavorable prognosis in terms of injuries and deaths.

Keywords: Diabetes Mellitus; Glicemic Control; Lifestyle.
\end{abstract}

\section{INTRODUÇÃO}

O Diabetes Mellitus (DM) é uma doença crônica não transmissível de grande incidência no Brasil e responsável por alta morbimortalidade ${ }^{(1,2)}$. É uma doença metabólica caracterizada por hiperglicemia e associadas a complicações, disfunções e insuficiência de vários órgãos ${ }^{(1,2-8)}$. Seus tratamento e controle exigem alterações de comportamento em relação à dieta, ingestão de medicamentos e o estilo de vida.

O controle adequado da glicemia, dos lipídios plasmáticos e da pressão arterial de pacientes com DM2 retarda ou previne o aparecimento das complicações micro e macrovasculares relacionadas à doença ${ }^{(8-13)}$. A manutenção desse controle associa- da ao controle dos lipídeos séricos, pressão arterial e peso corporal adequado deve ser um dos principais focos no tratamento dietoterápico destes pacientes $^{(14,15)}$. O exercício físico também deve fazer parte do tratamento do DM e seu papel é tão importante quanto a dieta e a medicação. Infelizmente, esta prática é pouco frequente, possivelmente devido à falta de compreensão e/ou motivação por parte dos pacientes e da equipe de saúde, pois a própria recomendação de exercícios por parte destes últimos continua deficiente ${ }^{(16-2)}$.

O manejo do diabetes deve ser feito dentro de um sistema hierarquizado de saúde, tendo em sua base o nível primário. Porém, a despeito do pre- 
conizado na atenção básica e das diversas campanhas de incentivo à prevenção e o controle da DM por meio de hábitos saudáveis de vida, os dados sugerem que os profissionais da saúde ainda enfrentam dificuldades em tornar efetivas as medidas de prevenção primária. O número de agravos decorrentes desta patologia também não diminuiu nos últimos anos prevalecendo elevada mortalidade, o que também sugere falha nos processos de cuidados assistenciais. O controle glicêmico é apontado como o principal fator de proteção para redução de agravos em diabéticos, mas as evidencias cientificas tem apontado que na atenção primaria tal objetivo tem sido difícil de alcançar. Todavia, dados locais sobre esta realidade não foram localizados. Deste modo, objetivou-se investigar os fatores associados à ausência de controle glicêmico em pacientes com DM tipo 2.

\section{MATERIAL E MÉTODOS}

Trata-se se um estudo de corte transversal conduzido com pacientes com diagnóstico de DM e que estavam em tratamento em unidades de saúde do Distrito Sanitário Cabula-Beiru (DSCB), Salvador, Bahia, no período de maio a julho de 2013. Foram incluídos todos os que estavam aguardando atendimento no período da coleta ou em busca de medicações na própria unidade e que aceitaram participar do estudo. O cálculo amostral foi realizado pela calculadora Winpepi (http://www.brixtonhealth.com/pepi4windows.html). Estimou-se um tamanho mínimo de amostra de 72 usuários considerando um erro amostral de $5 \%$, nível de confiança de $95 \%$, uma população de 400.000 habitantes para o DSCB e uma prevalência máxima de Diabetes Mellitus de 6\%. Em função de possíveis perdas ou recusas decidiu-se ampliar em mais de $50 \%$ o tamanho amostral.

Dados primários foram coletados e as entrevistas foram realizadas por profissionais de saúde e alunos dos cursos de saúde da Universidade do Estado da Bahia. Todos os pesquisadores foram de- vidamente treinados para a coleta com o intuito de otimizar a confiabilidade dos dados obtidos.

Um instrumento estruturado foi desenvolvido pelos autores do estudo contendo variáveis sociodemográficas, relativas a hábitos de vida, condições clínicas, e sobre a qualidade da assistência na atenção básica. Entre as variáveis sociodemográficas questionou-se a idade em anos completos e cor da pele, definida pelo entrevistador, categorizada de acordo com o IBGE, mas colapsadas em branca e pretos e pardos (http://www.ibge.gov.br/ home/estatistica/populacao/condicaodevida/indicadoresminimos/conceitos.shtm). A escolaridade foi definida em analfabeto, $1^{\circ}$ grau completo ou incompleto ou $2^{\circ}$ grau e/ou superior completo ou incompleto. A situação conjugal foi categorizada em vive com companheiro ou não. A renda familiar foi definida em menor ou maior que um salário mínimo.

Considerou-se ausência de controle glicêmico os valores iguais ou acima de $126 \mathrm{mg} / \mathrm{dl}$ obtidos por exame de glicose plasmática no sangue do paciente em jejum (http://bvsms.saude.gov.br/bvs/ publicacoes/consenso_bras_diabetes.pdf), e relatados pelos mesmos. Com relação aos hábitos de vida foram questionados se os mesmos fumavam atualmente (http://www.projetodiretrizes.org.br/ ans/diretrizes/tabagismo.pdf) e se bebiam mais que quatro - para mulheres, e cinco - para os homens, doses de bebida alcoólica em uma única ocasião e as respostas para estas questões variaram entre sim e não (http://bvsms.saude.gov.br/ bvs/publicacoes/relatorio_padroes_consumo_alcool.pdf). Considerou-se ativo fisicamente aquele que afirmou praticar atividade física de intensidade leve ou moderada por mais de trinta minutos em cinco ou mais dias na semana. O consumo de cinco ou mais porções diárias de frutas, hortaliças e cereais foi considerado como variável dicotômica.

No bloco referente aos dados clínicos do paciente questionou-se a presença de história familiar de diabetes, tratamento medicamentoso e presença de Hipertensão Arterial Sistêmica sendo estas foram categorizadas em sim e não. O Índice de 
Massa Corpórea (IMC) foi calculado dividindo-se o peso $(\mathrm{kg})$ pela altura $\left(\mathrm{m}^{2}\right)$. Posteriormente, este foi categorizado em abaixo do peso ou peso normal $\left(<24,9 \mathrm{~kg} / \mathrm{m}^{2}\right)$, e sobrepeso e obesidade para os demais.

Quanto às variáveis relativas à qualidade da atenção prestada questionou-se onde o paciente realizava a maioria das consultas e onde conseguia remédios para cuidar da doença, se na própria USF de abrangência, ou em outra unidade de saúde. A variável realização de consulta para controle da doença nos últimos seis meses foi considerada categórica, assim como se sai da consulta com a próxima já marcada e se algum profissional da equipe examinou os pés nos últimos seis meses. Foi também considerado como dicotômica saber se na unidade que frequenta tem grupos de apoio ou convivência.

O banco de dados foi confeccionado no programa Excel for Windows e em seguida realizado procedimentos de limpeza. Análises bivariadas foram realizadas com o intuito de identificar o conjunto de variáveis que mais se associaram com a ausência do controle glicêmico. A magnitude da associação entre as variáveis estudadas e a ausência de controle glicêmico foi estimada pelo cálculo da razão de chances (odds ratio, OR), adotando-se o intervalo de confiança a $95 \%$ (IC95\%) como medida de precisão. Posteriormente, foram realizadas análises multivariadas utilizando-se a regressão logística. A estratégia utilizada para a entrada dos blocos de variáveis foi do tipo forward (processo anterógrado). Permaneceram no modelo as variáveis que mostraram níveis de significância estatística, segundo um $p<0,10$. Utilizou- se o programa Stata (versão 12.0) para as análises. O projeto de pesquisa foi aprovado pela Plataforma Brasil/ CEP UNEB ( $N^{\circ} 241.434 / 2013$ ) em conformidade com as normas da Resolução 466/12 do Conselho Nacional de Ética em Pesquisa (CONEP).

\section{RESULTADOS}

Encontravam-se em tratamento nas unidades de saúde do Distrito Sanitário Cabula Beiru 127 diabéticos que preencheram adequadamente os critérios de elegibilidade. Dentre estes, 51 (40,2\%) apresentavam níveis glicêmicos considerados como não controlados, ou seja, acima de $126 \mathrm{mg} / \mathrm{dl}$. $\mathrm{Na}$ Tabela 1 encontram-se descritos as frequências $\mathrm{e}$ as análises bivariadas e multivariadas dos dados sociodemográficos e controle do nível glicêmico. As análises bivariadas não mostraram significância estatística, exceto para a associação entre ter renda familiar menor ou igual a um salário mínimo e ausência de controle glicêmico $(p=0,031)$. Ressalta-se que na multivariada esta variável permaneceu estatisticamente significante $(p=0,035)$ e o sexo, embora não tenha sido estatisticamente significante $(p<0,10)$ permaneceu no modelo para fins de ajustes.

(continua)

\begin{tabular}{ccccc}
\hline & \multicolumn{4}{c}{ AUSÊNCIA DE CONTROLE GLICÊMICO } \\
\cline { 2 - 3 } VARIÁVEIS & $\mathrm{N}(\%)$ & OR BRUTA & OR AJUSTADA & VALOR DE P \\
& $($ IC 95\%) & (IC 95\%) & \\
\hline
\end{tabular}

\section{Sexo}

Masculino

Feminino

Idade
$30(23,6)$

$97(76,4)$
1

$1,24(0,56-3,18)$
1

$1,21(0,68-6,45)$

0,195

\section{4 a 59}

$53(41,7)$

$60 e^{+}$

$74(58,3)$

1

$1,41(0,68-2,91)$
1

$1,52(0,61-3,72)$

0,346 


\begin{tabular}{|c|c|c|c|c|}
\hline \multirow{3}{*}{ VARIÁVEIS } & \multicolumn{3}{|c|}{ AUSÊNCIA DE CONTROLE GLICÊMICO } & \multirow{3}{*}{ VALOR DE P } \\
\hline & & OR BRUTA & OR AJUSTADA & \\
\hline & $\mathrm{N}(\%)$ & (IC 95\%) & (IC 95\%) & \\
\hline Brancos & $9(7,1)$ & 1 & & \\
\hline Pretos e pardos & $118(92,9)$ & $1,27(0,30-5,36)$ & $1,02(0,18-5,80)$ & 0,738 \\
\hline \multicolumn{5}{|l|}{ Situação conjugal } \\
\hline $\begin{array}{l}\text { Casados ou vivendo em união } \\
\text { estável }\end{array}$ & $56(44,1)$ & 1 & 1 & \\
\hline Vivendo sem companheiro & $71(55,9)$ & $1,24(0,60-2,51)$ & $1,61(0,64-4,80)$ & 0,556 \\
\hline \multicolumn{5}{|l|}{ Escolaridade } \\
\hline $2^{\circ}$ grau completo / superior & $31(24,4)$ & 1 & & \\
\hline Analfabeto $/ 1^{\circ}$ grau & $96(75,6)$ & $0,99(0,43-2,28)$ & $0,93(0,32-2,70)$ & 0,987 \\
\hline \multicolumn{5}{|l|}{ Renda familiar (em salários } \\
\hline \multicolumn{5}{|l|}{ mínimos) } \\
\hline$>1$ & $48(37,8)$ & 1 & & \\
\hline .1 & $79(62,2)$ & $2,53(1,10-5,90)$ & $2,94(1,18-8,05)$ & 0,031 \\
\hline
\end{tabular}

* Variáveis com valor de p. 0,10 integraram o modelo da regressão logística multivariada ajustado por renda e sexo.

Os diabéticos entrevistados tinham, mais frequentemente, hábitos de vida pouco saudáveis. Verificou-se que $79,5 \%$ indivíduos não praticavam atividade física de intensidade leve ou moderada por 30 minutos ou mais. Este fator se mostrou estatisticamente associado ao não controle glicêmico (OR=2,39; IC95\%:1,85-6,69), mesmo após o ajuste por sexo e renda no modelo multivariável (OR=2,14; IC95\%: 1,88-8,22) (Tabela 2).

Tabela 2 - Análise bivariada dos hábitos de vida dos portadores de Diabetes Mellitus vinculados a unidades de atenção primária de um distrito sanitário e ausência de controle glicêmico, Salvador, Bahia, 2013. N=127

(continua)

\begin{tabular}{|c|c|c|c|c|}
\hline \multirow[b]{2}{*}{ VARIÁVEIS } & \multicolumn{3}{|c|}{ AUSÊNCIA DE CONTROLE GLICÊMICO } & \multirow[b]{2}{*}{ VALOR DE } \\
\hline & N (\%) & $\begin{array}{c}\text { OR BRUTA } \\
\text { (IC 95\%) }\end{array}$ & $\begin{array}{c}\text { OR AJUSTADA } \\
\text { (IC 95\%) }\end{array}$ & \\
\hline \multicolumn{5}{|l|}{ Fuma atualmente } \\
\hline Não & $11(8,6)$ & 1 & & \\
\hline Sim & $116(91,4)$ & $\begin{array}{c}3,15(0,63- \\
14,82)\end{array}$ & $1,03(0,72-5,77)$ & 0,998 \\
\hline \multicolumn{5}{|c|}{ Consumo elevado de bebida nos } \\
\hline \multicolumn{5}{|c|}{ últimos trinta dias } \\
\hline Não & $100(78,7)$ & 1 & 1 & \\
\hline Sim & $27(21,3)$ & $1,65(0,65-4,12)$ & $1,96(0,53-7,25)$ & 0,311 \\
\hline
\end{tabular}


Tabela 2 - Análise bivariada dos hábitos de vida dos portadores de Diabetes Mellitus vinculados a unidades de atenção primária de um distrito sanitário e ausência de controle glicêmico, Salvador, Bahia, 2013. N=127

(conclusão)

\begin{tabular}{|c|c|c|c|c|}
\hline \multirow[b]{2}{*}{ VARIÁVEIS } & \multicolumn{3}{|c|}{ AUSÊNCIA DE CONTROLE GLICÊMICO } & \multirow[b]{2}{*}{ VALOR DE P } \\
\hline & $N(\%)$ & $\begin{array}{c}\text { OR BRUTA } \\
\text { (IC 95\%) }\end{array}$ & $\begin{array}{c}\text { OR AJUSTADA } \\
\text { (IC 95\%) }\end{array}$ & \\
\hline Sim & $34(26,7)$ & 1 & 1 & \\
\hline Não & $93(73,3)$ & O,61 (O,27 - 1,37) & O,73 (O,26 - 2,O2) & 0,549 \\
\hline
\end{tabular}

\section{Pratica alguma atividade física}

leve/moderada por trinta

minutos ou mais

$\begin{array}{lcccc}\text { Sim } & 26(20,5) & 1 & 1 & \\ \text { Não } & 101(79,5) & 2,14(1,83-5,51) & 2,39(1,85-6,69) & \mathbf{0 , 0 4 9}\end{array}$

* Variáveis com valor de p· 0,10 integraram o modelo da regressão logística multivariada ajustado por renda, sexo e atividade física.

$\mathrm{Na}$ Tabela 3 descrevem-se as frequências, as razões de chance brutas e ajustadas entre ausência de controle glicêmico e variáveis clinicas. As variá- veis deste bloco não se associaram de modo significante com a ausência de controle glicêmico.

Tabela 3 - Análise bivariada de características clínicas dos portadores de Diabetes Mellitus vinculados a unidades de atenção primária de um distrito sanitário e ausência de controle glicêmico, Salvador, Bahia, 2013. N=127

\begin{tabular}{ccccc}
\hline \multirow{3}{*}{ VARIÁVEIS } & \multicolumn{3}{c}{ AUSÊNCIA DE CONTROLE GLICÊMICO } & OR A OR DE P \\
\cline { 2 - 4 } & $\mathrm{N}(\%)$ & $\begin{array}{c}\text { OR BRUTA } \\
\text { (IC 95\%) }\end{array}$ & $\begin{array}{c}\text { OR AJUSTADA } \\
\text { (IC 95\%) }\end{array}$ & VALOR DE \\
\hline
\end{tabular}

\section{Índice de Massa Corpórea}

Abaixo do peso ou normal

Sobrepeso/obesidade
$34(26,7)$

$93(73,2)$
$0,61(0,27-1,37)$

0,611

$\mathrm{O}, 71(0,2 \mathrm{O}-$

2,56)

\section{História familiar de diabetes}

Sim

Não
$26(20,5)$

$101(79,5)$
1

$0,55(0,17-1,75)$
0,266

\section{Tipo de Diabetes}

1

$$
81(96,1)
$$

1

$5(3,9)$

$1,91(0,12-11,1)$

$1,19(0,12-11,9)$

0,878

\section{Tratamento medicamentoso}

$\begin{array}{lccc}\text { Sim } & 108(85,0) & 1 & 1 \\ \text { Não } & 19(15,0) & 1,69(0,45-6,26) & 1,50(0,38-5,91)\end{array}$

0,561

\section{Hipertensão Arterial}

$\begin{array}{lrcr}\text { Não } & 85(66,9) & 1 & 1 \\ \text { Sim } & 42(33,1) & 2,22(0,82-5,97) & 2,15(0,77-1\end{array}$

1

* Variáveis com valor de p. 0,10 integraram o modelo da regressão logística multivariada ajustado por renda, sexo, atividade física, tempo de tratamento de diabetes, última consulta, dor no peito e retinopatia. 
Análises bivariadas e multivariadas sobre a qualidade da assistência aos portadores de Diabetes Mellitus em unidades de saúde de atenção primária e ausência do controle glicêmico, bem como as razões de chance brutas e ajustadas pelas variáveis do bloco qualidade da assistência encontram-se dispostas na Tabela 4. Essas variáveis não se associaram de forma significativa com o controle glicêmico antes ou após ajuste pelas variáveis do bloco.

Tabela 4 - Análise bivariada de características relativa à qualidade da assistência aos portadores de Diabetes Mellitus vinculados a unidades de atenção primária de um distrito sanitário e ausência de controle glicêmico,

\begin{tabular}{|c|c|c|c|c|}
\hline \multirow[b]{2}{*}{ VARIÁVEIS } & \multicolumn{3}{|c|}{ AusÊNCIA DE CONTROLE GLICÊMICO } & \multirow[b]{2}{*}{ VALOR DE P } \\
\hline & $\mathrm{N}(\%)$ & $\begin{array}{l}\text { OR BRUTA } \\
\text { (IC 95\%) }\end{array}$ & $\begin{array}{l}\text { OR AJUSTADA } \\
\text { (IC 95\%) }\end{array}$ & \\
\hline \multicolumn{5}{|l|}{ Onde fez a maioria das consultas } \\
\hline \multicolumn{5}{|c|}{ para cuidar da Diabetes nos últimos } \\
\hline \multicolumn{5}{|c|}{6 meses } \\
\hline Na USF do território & $68(53,5)$ & 1 & 1 & \\
\hline Em outra unidade de saúde & $59(46,5)$ & $0,59(0,22-1,22)$ & $0,61(0,24-1,83)$ & 0,381 \\
\hline \multicolumn{5}{|l|}{ Sai da consulta com a próxima } \\
\hline \multicolumn{5}{|l|}{ marcada } \\
\hline Sim & $28(22,1)$ & 1 & 1 & \\
\hline Não & $99(77,9)$ & $1,87(0,73-4,75)$ & $0,62(0,18-2,06)$ & 0,438 \\
\hline \multicolumn{5}{|c|}{ Profissional da equipe examinou os } \\
\hline \multicolumn{5}{|c|}{ pés nos últimos seis meses } \\
\hline Sim & $34(26,7)$ & 1 & 1 & \\
\hline Não & $93(73,3)$ & $0,83(0,32-2,18)$ & $0,85(0,22-3,81)$ & 0,822 \\
\hline \multicolumn{5}{|l|}{$\begin{array}{l}\text { Onde, na maioria das vezes, } \\
\text { consegue o remédio para DM }\end{array}$} \\
\hline Na própria unidade & $67(52,8)$ & 1 & 1 & \\
\hline Outras unidades & $60(47,2)$ & $0,55(0,23-1,27)$ & $\begin{array}{c}0,62(0,20- \\
1,83)\end{array}$ & 0,381 \\
\hline \multicolumn{5}{|l|}{$\begin{array}{l}\text { Frequenta grupos de apoio/ } \\
\text { convivência nesta unidade }\end{array}$} \\
\hline Sim & $21(16,5)$ & 1 & 1 & \\
\hline Não & $106(83,5)$ & $1,17(0,35-3,21)$ & $1,15(0,21-6,15)$ & 0,862 \\
\hline
\end{tabular}

Salvador, Bahia, 2013. N=127 * Variáveis com valor de p. O,10 integraram o modelo da regressão logística multivariada ajustado por renda, sexo, atividade física,
tempo de tratamento de diabetes, última consulta, dor no peito e retinopatia.

Na Tabela 5 apresentam-se as variáveis do modelo final de regressão logística. Na análise multivariada, a ausência de controle glicêmico se mostrou associada com não praticar atividade física leve a moderada regularmente $(\mathrm{OR}=2,14 ; \mathrm{IC} 95 \%: 1,88$ 2,22), mesmo após ajuste por sexo, renda, relativas a hábitos de vida e qualidade da assistência. 
Tabela 5 - Odds ratios bruto e ajustado para ausência de controle do nível glicêmico de portadores de Diabetes Mellitus vinculados a unidades de atenção primária de um distrito sanitário, segundo as variáveis que permaneceram no modelo da regressão logística multivariada. Salvador, Bahia, 2013.

\begin{tabular}{lccc}
\hline \multirow{2}{*}{ VARIÁVEL } & \multicolumn{2}{c}{ AUSÊNCIA DE CONTROLE GLICÊMICO } & \\
\cline { 2 - 3 } & $\begin{array}{c}\text { OR BRUTO (IC } \\
\mathbf{9 5 \%}\end{array}$ & $\begin{array}{c}\text { OR AJUSTADO (IC } \\
\text { 95\%) }\end{array}$ & VALOR DE P \\
\hline Sexo & $1,24(0,56-3,18)$ & $2,10(0,68-6,45)$ & 0,195 \\
Renda & $2,53(1,10-5,90)$ & $2,18(0,82-5,78)$ & 0,117 \\
$\begin{array}{l}\text { Não praticar atividade física leve/ } \\
\text { moderada regularmente }\end{array}$ & $2,69(1,83-5,51)$ & $2,14(1,88-8,22)$ & 0,051 \\
\hline
\end{tabular}

* Variáveis com valor de p· o,10 integraram o modelo da regressão logística multivariada ajustado por renda, sexo, atividade física, tempo de tratamento de diabetes, última consulta, dor no peito e retinopatia.

\section{DISCUSSÃO}

A prevalência do não controle glicêmico observado neste estudo foi considerada como elevada, $(40,2 \%)$ e se assemelha àquela relatada por Franco Junior e outros, ${ }^{(20)}$ em São Bernardo do Campo, São Paulo $(46,7 \%)$, embora os autores citados tenham realizado a pesquisa em unidades do setor privado. Os mesmos relataram que esperavam melhor controle glicêmico nesta população considerando a oferta de serviços de melhor qualidade. Ausência de controle glicêmico foram reportadas também por Panarotto e outros, ${ }^{(13)}$ tanto em unidades de serviços públicos de atenção primária $(191,5 \pm 88,5 \mathrm{mg} / \mathrm{dl})$ quanto em unidades de serviços privados $(180,4 \pm 75,1 \mathrm{mg} / \mathrm{dl}) \quad(p=0,47)$. É possível que, isoladamente, o sistema de saúde no qual o indivíduo seja tratado não se comporte como determinante do controle glicêmico em pacientes com DM2. Outrossim, variáreis relacionadas ao acesso do paciente às consultas, como o controle inicial do diabetes e a frequência às mesmas, podem ser fatores importantes e reforça a necessidade de educação continuada em saúde. ${ }^{(24)}$ Assunção e outros, (21) identificaram prevalência de $50 \%$ no controle glicêmico em pacientes diabéticos atendidos em centros de cuidados primários à saúde. Concluíram que aqueles que faziam uso de medicações eram os que menos tinham controle dos níveis glicêmicos. Deve-se levar em consideração que, isoladamente, o uso das medicações pode não atingir os objetivos terapêuticos desejados e que é fundamental a associação com programas efetivos de educação em saúde.

Grande parte dos pacientes entrevistados no estudo atual negaram a prática regular de atividade física intensa ou moderada. Pode ter contribuído para este resultado o local de moradia, áreas com ladeiras, sem espaço para a prática de caminhadas ou ciclismo. Tais resultados são corroborados por Paiva e outros, ${ }^{(19)}$ que investigando a assistência prestada a hipertensos e diabéticos residentes em Francisco Morato, SP, relataram que $75 \%$ dos indivíduos referiram não ter o habito de praticar atividade física por residir em ruas íngremes, por falta de tempo ou ainda por dores ou cansaço. De modo semelhante Villas-Boas e outros, ${ }^{(22)}$ evidenciaram baixa adesão à dieta e ao exercício físico. Salientaram ainda que as mudanças no estilo de vida representam uma grande adversidade para as pessoas, principalmente quando se trata de fazer restrições alimentares e praticar exercícios físicos.

A dificuldade dos pacientes em adquirir um estilo de vida saudável no tratamento do DM também foi evidenciada por Paiva e outros, ${ }^{(19)}$ que encontraram que apenas $32,8 \%$ dos usuários entrevistados mostraram ter uma dieta adequada e $75,2 \%$ não tinham o hábito de praticar atividade física. Em 
estudos sobre controle dos fatores de risco para diabéticos, os pesquisadores frequentemente discutem sobre o papel da atividade física no controle glicêmico ${ }^{(15-17,20,22,23)}$. Vancea e outros ${ }^{(17)}$ afirmaram que um programa de exercício físico estruturado de intensidade moderada é capaz de provocar uma redução no IMC já a partir da oitava semana de treinamento. Observaram ainda que ao aumentar a frequência de atividades físicas para cinco vezes por semana é possível ter efeitos adicionais, como redução na circunferência abdominal, na média das glicemias capilares e nas glicemias de jejum e pós-prandial.

No presente estudo observou-se um grande número de pacientes com sobrepeso ou obesidade grau I. Este resultado sugere um importante obstáculo enfrentado em relação à mudança de estilo de vida e alimentação.(7) Estes dados também apontam para uma plausível falha no acompanhamento multiprofissional da atenção primária e chama a atenção para o risco cardíaco que estes pacientes podem apresentar futuramente. Muitos entrevistados, no presente estudo, associaram a falta de renda à má alimentação alegando renda insuficiente para comprar frutas, legumes e carnes frescas e dando preferência a alimentos industrializados e mais baratos.

Alguns pontos negativos em relação à qualidade da assistência foram detectados no presente estudo. É possível supor que houve falhas na assistência ofertada no período da pesquisa a despeito destas não terem se associado significativamente com a ausência de controle glicêmico, tal como estimado por Mielczarski e outros, ${ }^{(25)}$ que no RS, observaram que $64,9 \%$ dos indivíduos não foram informados sobre os cuidados com os pés. De modo semeIhante Paiva e outros, ${ }^{(19)}$ identificaram que $53,1 \%$ dos usuários do PSF referiram nunca participado de aulas, grupos ou palestras relacionados com sua patologia.

Como vantagens deste trabalho destaca-se a vinculação ao PRÓ-Saúde/Pet-Saúde e apoio dos profissionais do DSCB.

\section{CONCLUSÕES}

A proporção de diabéticos tipo 2 atendidos nas unidades de saúde do DSCB que não controlam os níveis glicêmicos é elevada e pode contribuir para o aumento das complicações cardiovasculares e para um prognóstico desfavorável em termos de agravos e óbitos. De acordo com o Plano de Reorganização da Atenção à Hipertensão Arterial e ao Diabetes Mellitus as intervenções propostas para diabéticos em atendimento ambulatorial na atenção básica devem preconizar o manejo de risco multifatorial. Assim, o combate ao excesso de peso corporal e ao sedentarismo, e a baixa frequência de ações de educação em saúde são atividades que independem da renda familiar mensal e que devem ser incorporadas na atenção básica como preconiza o Sistema Único de Saúde. Novos estudos devem ser conduzidos no sentido de evidenciar alterações proporcionadas por mudanças no cenário político local.

\section{REFERÊNCIAS}

1. Miranzi SSC, Ferreira FS, Iwamoto HH, Pereira GA, Miranzi MAS. Qualidade de vida de indivíduos com diabetes mellitus e hipertensão acompanhados por uma equipe de saúde da família. Texto Contexto Enferm. 2008;17(4):6729.

2. Ministério da Saúde. Diabetes Mellitus Cadernos de Atenção Básica n 16. Brasília 2006.

3. Instituto Brasileiro de Estatística (IBGE) - Relatório populacional dos municípios brasileiros; 2008. [Citado 2013 ago 16]. Disponível em: www.ibge.gov.br

4. Organização Mundial da Saúde (OMS); 2003. Screening for type 2 diabetes, report of a World Health Organization and International Diabetes Federation meeting.

5. Lyra R, Oliveira M, Lins D, Cavalcanti N. Prevenção do Diabetes Mellitus Tipo 2. Arq. Bras. Endocrinol. Metab. 2006;50(2):239-49.

6. Pitta GBB, Castro AA, Soares AMM, Maciel CJJ, Silva JDM, VMT Muniz, Asmar SB. Perfil 
dos Pacientes Portadores de Pé Diabético Atendidos no Hospital Escola José Carneiro e na Unidade de Emergência Armando Lages. J. Vas. Br. 2005;4(1):5-10.

7. Gomes MB, Giannella DN, Mendonça E, Tambascia MA, Fonseca RM, Réa RR et al. Prevalência de sobrepeso e obesidade em pacientes com diabetes mellitus do tipo 2 no Brasil: estudo multicêntrico nacional. Arq. Bras. Endocrinol. Metab. 2006;50(1):136-44.

8. Schefel RS, Bortolanza M, Weber CS, Costa LA, Canani LH, SantosKG et al. Prevalência de complicações micro e macrovasculares e de seus fatores de risco em pacientes com diabetes melito do tipo $2 \mathrm{em}$ atendimento ambulatorial. Rev. Assoc. Med. Bras. 2004;5O(3): 263-7.

9. Rocha RM, Zanetti ML, Santos MA. Comportamento e conhecimento: fundamentos para prevenção do pé diabético. Acta Paul Enferm 2009;22(1):17-23.

10. Ferreira MJ, Sampaio FMO, Coelho JMS, Almeida NMGS. Perfil audiológico de pacientes com diabetes mellitus tipo II. Artigo Original. Rev. Soc. Bras. Fonoaudiol. 2007; 12(4):292-7.

11. Haddad AE, Campos FE, Freitas MSBF, Brenelli SL, Passarella TM, Ribeiro TCV. Programa de educação pelo trabalho para a saúde- PetSaúde. Cadernos ABEM. 2009;5:6-12.

12. Plano de reorganização da atenção à hipertensão arterial e ao diabetes mellitus: hipertensão arterial e diabetes mellitus / Departamento de Ações Programáticas Estratégicas. - Brasília: Ministério da Saúde; 2001.

13. Panarotto D, Träsel HAV, Oliveira MS, Gravina LBA, Teles R. Controle glicêmico nos setores público e privado. Arq Bras Endocrinol Metab. 2009;53(6):733-40.

14. Silva FM, Steemburgo T, Azevedo MJ, Mello VD. Papel do índice glicêmico e da carga glicêmica na prevenção e no controle metabólico de pacientes com diabetes mellito tipo 2. Arq Bras Endocrinol Metabol 2009;53(5):560-71.

15. Zanuso S, Jimenez A, Pugliese G, Corigliano $G$, Balducci $S$. Exercise for the management of type 2 diabetes: a review of the evidence. Acta Diabetol. 2010;47(1):15-22.
16. Moro ARP, Silva FC, lop RR, Filho PJBG. Efeito do treinamento combinado e aeróbio no controle glicêmico no diabetes tipo 2. Fisioter Mov. 2012;25(2):399-409.

17. Vancea DMM, Vancea JN, Pires MIF, Reis MA, Moura RB, Dib SA. El Efecto de la Frecuencia del Ejercicio Físico en el Control Glucémico y Composición Corporal de Diabéticos Tipo 2. Arq Bras Cardiol 2009;92(2):23-29.

18. McLellan KCP, Barbalho SM, Cattalini M, Lelario AC. Diabetes Mellitus do tipo 2, síndrome metabólica e modificação no estilo de vida. Rev. Nutr. 2007;2O(5):515-524.

19. Paiva DCP, Bersusa AAS, Escuder MML. Avaliação da assistência ao paciente com diabetes e/ou hipertensão pelo Programa Saúde da Família do Município de Francisco Morato, São Paulo, Brasil. Cad. Saúde Pública, Rio de Janeiro 2006;22(2):377-385.

2O. Franco Júnior AJA, Heleno MGV, Lopes AP. Qualidade de vida e controle glicêmico do paciente portador de diabetes mellitus tipo 2 . Rev. Psicol. e Saúde 2013;5(2):102-108.

21. Assunção MCF, Santos IS, Valle NCJ. Blood glucose control in diabetes patients seen in primary health care centers. Rev Saúde Pública 2005;39(2):183-190.

22. Villas-Boas LCG, Foss MC, Freitas MCF, Torres HC, Monteiro LZ, Pace AM. Adesão á dieta e ao exercício físico das pessoas com Diabetes Mellitus. Texto Contexto Enferm 2O11; 2O(2): 272-9.

23. Unaise AH, Manzar D, Razar S, Shareef MY, Hussain ME. Resistance Training Leads to Clinically Meaningful Improvements in Control of Glycemia and Muscular Strength in Untrained Middle-aged Patients with type 2 Diabetes Mellitus. North Americ Jour of Medical Sciences. 2012;4(8):336-343.

24. Torres HC, Franco LJ, Stradioto MA, Hortale VA, Shall VT. Avaliação estratégica de educação em grupo e individual no programa educativo em diabetes. Rev Saúde Pública 2009;43(2):291-8.

25. Mielczarski RG, Costa JSD, Olinto MTA. Epidemiologia e organização de serviços de saúde: diabetes mellitus numa comunidade de Porto Alegre. Ciência \& Saúde Col 2O12; 17(1):71-78. 